Published in final edited form as:

React Oxyg Species (Apex). 2016 ; 1(1): 9-21. doi:10.20455/ros.2016.803.

\title{
Defining ROS in Biology and Medicine
}

\author{
Robert Li ${ }^{1,2,3,4}$, Zhenquan Jia ${ }^{4}$, and Michael A. Trush ${ }^{5}$ \\ ${ }^{1}$ Departments of Pharmacology and Pharmaceutical Sciences, Campbell University, Buies Creek, \\ NC 27506, USA \\ ${ }^{2}$ Virginia Tech-Wake Forest University School of Biomedical Engineering and Sciences, \\ Blacksburg, VA 24061, USA \\ ${ }^{3}$ Department of Biomedical Sciences and Pathobiology, Virginia Tech, Blacksburg, VA 24061, \\ USA \\ ${ }^{4}$ Department of Biology, University of North Carolina, Greensboro, NC 27412, USA \\ ${ }^{5}$ Department of Environmental Health Sciences, The Johns Hopkins University, Baltimore, MD \\ 21205, USA
}

\begin{abstract}
Utilization of molecular oxygen by aerobic organisms inevitably results in the formation of a number of oxygen-containing reactive species that are collectively known as reactive oxygen species (ROS). ROS play important roles in both physiology and pathophysiology of aerobic life. The field of 'ROS biology and medicine' deals with the involvement of ROS and related species in contemporary biology and medicine. The purpose of this article is to survey common terms and concepts in ROS biology and medicine. It also introduces the 'ROS paradigm' so as to provide a conceptual framework for understanding the rapidly evolving field of ROS biology and medicine.
\end{abstract}

\section{Keywords}

Oxidative stress; Reactive oxygen species; Redox signaling; ROS paradigm

\section{ROS BIOLOGY AND MEDICINE}

ROS biology and medicine is a relatively new field. To define it, we need to first consider the definitions of biology and medicine. The word biology is defined as the science of life and living things (e.g., plants and animals), studying their structure, function, growth, origin, evolution, and distribution. On the other hand, the word medicine refers to the science or practice of the diagnosis, treatment, and prevention of disease. Modern medicine developed primarily as a result of our profound understanding of human and animal biology at the molecular and cellular levels. ROS biology and medicine may be defined as a field that studies the biological effects of ROS and related species as well as antioxidants with an emphasis on the involvement of these molecular entities in health and disease. 


\section{ROS AND RELATED TERMS}

\subsection{ROS}

ROS is a term frequently encountered in biology and medicine. This term can be simply defined as oxygen-containing reactive species. It is a collective term to include superoxide $\left(\mathrm{O}_{2}{ }^{\mathbf{}}\right)$, hydrogen peroxide $\left(\mathrm{H}_{2} \mathrm{O}_{2}\right)$, hydroxyl radical $\left(\mathrm{OH}^{\mathbf{*}}\right)$, singlet oxygen $\left({ }^{1} \mathrm{O}_{2}\right)$, peroxyl radical (LOO'), alkoxyl radical (LO"), lipid hydroperoxide (LOOH), peroxynitrite (ONOO $\left.{ }^{-}\right)$, hypochlorous acid $(\mathrm{HOCl})$, and ozone $\left(\mathrm{O}_{3}\right)$, among others [1]. Although $\mathrm{ROS}$ is a widely used term in biology and medicine to describe oxygen-containing reactive species, other alternative terms also exist in the literature, which include reactive oxygen metabolites (ROMs), reactive oxygen intermediates (ROIs), and oxygen radicals. Among these various terms, ROS is most commonly used (Table 1).

\subsection{Oxygen Radicals}

Among the ROS listed above in Section 2.1, some contain unpaired electrons, and thus belong to free radicals. They are hence also called oxygen radicals or oxygen free radicals. The term free radical is defined as any chemical species capable of independent existence that contains one or more unpaired electrons. An unpaired electron refers to the one that occupies an atomic or molecular orbital by itself. Examples of oxygen radicals include superoxide, hydroxyl, peroxyl, and alkoxyl radicals.

On the other hand, several ROS do not contain any unpaired electrons, and as such, they are not free radicals. Examples of the non-radical ROS include hydrogen peroxide, peroxynitrite, hypochlorous acid, and ozone. As illustrated in Figure 1, singlet oxygen can exist in two states: the delta state $\left({ }^{1} \Delta_{\mathrm{g}}\right)$ and sigma state $\left({ }^{1} \Sigma_{\mathrm{g}}+\right) .\left({ }^{1} \Sigma_{\mathrm{g}}+\right)^{1} \mathrm{O}_{2}$ is a free radical because it contains two unpaired electrons, whereas $\left({ }^{1} \Delta_{\mathrm{g}}\right)^{1} \mathrm{O}_{2}$ is a non-radical. Due to its highly unstable nature, $\left({ }^{1} \sum_{\mathrm{g}}+\right)^{1} \mathrm{O} 2$ is generally regarded to lack any biological significance. Thus, in ROS biology and medicine, the term singlet oxygen, if not specified, usually refers to the ${ }^{1} \Delta_{\mathrm{g}}$ state.

\subsection{Reactive Nitrogen Species}

Similar to ROS, the term reactive nitrogen species (RNS) has been coined to include nitric oxide ( $\left.\mathrm{NO}^{-}\right)$, peroxynitrite, nitrogen dioxide radical $\left(\mathrm{NO}_{2}{ }^{-}\right)$, and other oxides of nitrogen or nitrogen-containing reactive species. Because RNS are almost exclusively oxygencontaining species, by definition, they may also be classified as ROS. For example, peroxynitrite is classified as either an ROS or an RNS.

\subsection{Reactive Chlorine Species}

The term reactive chlorine species (RCS) refers to chlorine-containing reactive species, with hypochlorous acid $(\mathrm{HOCl})$ as a prototype. As compared to ROS and RNS, the term RCS is less frequently used in biology and medicine (Table 1).

\subsection{Reactive Oxygen and Nitrogen Species}

As noted earlier, the RCS hypochlorous acid and the RNS peroxynitrite are also classified into the ROS category. Indeed, the ROS category comprises the most commonly 
encountered reactive species in biology and medicine. Due to the increasingly recognized biological effects of nitric oxide and related nitrogen-containing species, the term RNS has been becoming widely used in the biomedical literature. Frequently, the compound term 'reactive oxygen and nitrogen species (ROS/RNS)' is employed to refer to a group of ROS and RNS. Nevertheless, because all biologically relevant RNS are exclusively also oxygencontaining reactive species (and hence can be called ROS by definition), and for simplicity, the term ROS is used throughout the article to include both ROS and RNS.

\subsection{Electrophiles}

ROS are reactive species capable of causing damage to biomolecules, including proteins, lipids, and nucleic acids, leading to cell and tissue injury. Reactions of these reactive species with biomolecules also generate a large array of secondary electrophilic products (also known as electrophiles). These include $\alpha, \beta$-unsaturated aldehydes, $\omega-6$ and $\omega-3$ unsaturated fatty acids, as well as nitro-fatty acids. The term electrophile refers to an electron-deficient chemical species that undergoes covalent reactions by accepting an electron pair from an electron-rich biomolecule (also known as a nucleophile).

Electrophilic species can also be derived from biotransformation of xenobiotics [2, 3]. The term xenobiotic can be defined as any substance that does not occur naturally in the human body. Indeed, formation of electrophiles is a major mechanism of chemical toxicity. While high levels of electrophiles, especially a, $\beta$-unsaturated aldehydes, cause overt cell and tissue injury, these electrophilic species at non-cytotoxic levels can interfere with cell signaling [4, 5]. In this context, the endogenously formed electrophilic 8 -nitroguanosine $3^{\prime}, 5^{\prime}$-cyclic monophosphate acts as a second messenger for nitric oxide-mediated signal transduction (also see Section 5) [6, 7].

\section{ANTIOXIDANTS AND RELATED TERMS}

\subsection{Antioxidants}

Mammals including humans have evolved a series of antioxidant defenses to protect vital biomolecules from ROS and related species-mediated damage. In addition, a number of components derived from dietary sources also possess antioxidant activities in biological systems. The term antioxidant can be defined literally as any substance that can prevent, reduce, or repair the ROS-induced damage of a target biomolecule. In ROS biology and medicine, the target molecules usually include proteins, lipids, and nucleic acids, among others.

There are three major modes of action for antioxidants: (i) antioxidants that directly scavenge ROS already formed; (ii) antioxidants that inhibit the formation of ROS from their cellular sources; and (iii) antioxidants that remove or repair the damage or modifications caused by ROS.

There are many different kinds of antioxidants in biology and medicine, and they are classified in various ways. For example, superoxide dismutase is an endogenous antioxidant enzyme, whereas vitamin $\mathrm{C}$ is a widely known antioxidant derived from a variety of dietary sources. 


\subsection{Phase 2 Enzymes or Proteins}

Phase 1 and phase 2 (also written as phase I and Phase II) reactions are related to biotransformation (also known as metabolism) of xenobiotics. Phase 1 biotransformation reactions include oxidation, reduction, and hydrolysis. Phase 2 biotransformation involves primarily conjugation reactions, such as conjugation with endogenous cellular ligands (e.g., glutathione and glucuronic acid). Glutathione S-transferase and UDP-

glucuronosyltransferase catalyze conjugation with glutathione and glucuronic acid, respectively. These enzymes along with many others involved in phase 2 biotransformation reactions of xenobiotics are classically referred to as phase 2 proteins or enzymes. Recently, the term phase 2 proteins is expanded to include not only the above xenobiotic-conjugating enzymes, but also NAD(P)H:quinone oxidoreductase, epoxide hydrolase, dihydrodiol dehydrogenase, $\gamma$-glutamylcysteine ligase, heme oxygenase-1, leukotriene B4 dehydrogenase, aflatoxin B1 dehydrogenase, and ferritin [8]. Some of the above phase 2 proteins, such as $\gamma$-glutamylcysteine ligase, heme oxygenase-1, and ferritin are typically classified as antioxidants. As such, the compound term 'antioxidative/phase 2 proteins' is frequently encountered in biology and medicine. Indeed, many phase 2 proteins possess significant antioxidant activities.

\subsection{Antioxidative/Anti-inflammatory Defenses}

ROS and related species are mediators of inflammatory responses. In addition, ROS also activate cell signaling, augmenting production and release of proinflammatory cytokines, thereby perpetuating the inflammatory responses. Well-controlled inflammatory responses are part of our body's innate immunity, protecting against invading pathogens. However, overstimulated or prolonged inflammatory responses cause tissue injury and, as such, constitute a major pathophysiological mechanism of a wide variety of human diseases [9]. Due to the fact that ROS and inflammation are intimately intertwined $[10,11]$, many antioxidant enzymes or proteins, as well as non-protein antioxidants also possess antiinflammatory activities [12]. Hence, the compound term 'antioxidative/anti-inflammatory defenses' is sometimes used to describe these antioxidative molecules. In this context, nuclear factor E2-related factor 2 (Nrf2), a central regulator of antioxidant gene expression, also possesses anti-inflammatory function in diverse experimental systems [13]. The antiinflammatory action of Nrf2 signaling may also result from the upregulation of novel cytoprotective proteins and factors.

\section{OXIDATIVE STRESS AND RELATED TERMS}

\subsection{Oxidative Stress}

Oxidative stress is an important concept in biology and medicine. The term, initially coined in 1985 by Helmut Sies, refers to a condition where the levels of ROS significantly overwhelm the capacity of antioxidant defenses, leading to potential damage in a biological system [see ref. 1 in 13a]. Oxidative stress condition can be caused by either increased ROS formation or decreased activity of antioxidants or both in a biological system. Oxidative stress condition is associated with oxidative damage to biomolecules, including proteins, lipids, and nucleic acids. Moderate oxidative stress may cause cell dysfunction and altered behavior (e.g., accelerated senescence, abnormal proliferation, dysregulated inflammatory 
responses, and cell tumorigenesis), whereas overt oxidative stress usually causes cell death (e.g., oncosis, apoptosis, and autophagy).

Oxidative stress is a significant contributor to various pathophysiological processes. Figure 2 illustrates the concept of oxidative stress in ROS biology and medicine. It should be borne in mind that not any increases in ROS levels in a biological system are associated with injury. Under certain circumstances, small transient increases in ROS levels can be employed as a signaling mechanism, leading to physiological cellular responses (see Section 5).

\subsection{Nitrative Stress}

Analogous terms have been coined to describe the conditions associated with increased levels of RNS. For example, the term nitrative stress is defined as a condition under which the levels of RNS significantly overwhelm the capacity of RNS-detoxification mechanisms in a biological system. Nitrative stress condition is associated with nitration of biomolecules, leading to cell and tissue injury. Nitration refers to addition of a nitro $\left(-\mathrm{NO}_{2}\right)$ group to a compound. The RNS peroxynitrite readily causes nitrative stress in biological systems [14, $15]$.

\subsection{Nitrosative Stress}

Nitrosative stress is defined as a condition induced by nitric oxide (NO") or related species, leading to the formation of nitrosylation of critical protein cysteine thiols (S-nitrosylation) and metallocofactors of proteins. Nitrosylation refers to the addition of a nitroso (-NO) group to a thiol group or a redox active metal ion center of a protein. While dysregulation of nitrosylation is associated with a number of pathophysiological conditions, well-controlled nitrosylation plays an important role in cell signal transduction and provides a mechanism of redox-based physiological regulation in both animals and plants [16, 17].

\section{REDOX SIGNALING AND RELATED TERMS}

\subsection{General Considerations}

A number of terminologies of basic chemistry are also frequently used in ROS biology and medicine. These include oxidation, reduction, redox, as well as oxidant and reductant. Oxidation refers to loss of one or more electrons by an atom or a molecule. Reduction refers to gain of one or more electrons by an atom or a molecule. The term redox refers to reduction-oxidation. Redox biology is the study of oxidation-reduction processes associated with living things. In a chemical reaction, if chemical A oxidizes chemical B (another way of saying this is that chemical B reduces chemical A), then chemical A is an oxidant, chemical B a reductant for that particular reaction. In biological systems, many reductants act as antioxidants to detoxify ROS via either directly scavenging ROS or serving as electron donors for antioxidant enzymes. As described next, some of the cellular reductants also constitute redox metabolome, which interacts with redox proteome to impact cellular redox signaling. 


\subsection{Redox Proteome and Redox Metabolome}

The redox proteome is a collective term referring to components of the proteome that undergo reversible redox reactions and those modified irreversibly by ROS or related species [18]. Although many amino acids (e.g., tryptophan, tyrosine, and arginine) and the peptide backbone react with ROS, only three of the amino acids (cysteine, methionine, and selenocysteine) undergo reversible redox reactions. The redox proteome interacts with the redox metabolome, a redox-active subset of the metabolome, with NADPH/NADP ${ }^{+}, \mathrm{GSH} /$ GSSG (reduced form of glutathione/oxidized glutathione), and cysteine/cystine being specifically relevant to the redox proteome [18]. As mentioned above, the cellular reductants, such as NADPH, cysteine, and GSH can directly scavenge ROS (for cysteine and GSH) or serve as cofactors for antioxidant enzymes (for NADPH and GSH). The interactions between redox proteome, redox metabolome, and ROS form the basis of cellular redox signaling, an important concept that has emerged in recent years.

\subsection{Redox Signaling}

Before defining redox signaling, let's first consider the definition of cell signaling. Cell signaling is also known as cell signal transduction.

5.3.1. Cell Signaling: The Three Steps-Essential to the survival of every cell is to monitor the extracellular (as well as intracellular) environment, process the information it gathers, and respond accordingly. The concept of cell signaling defines the ability of cells to detect changes in their environment to generate an appropriate physiological response upon information processing. Cell signaling upon extracellular stimulation typically involves the following three major steps (Figure 3): (i) binding of the extracellular stimulus (e.g., a cytokine released by an adjacent cell) to a receptor protein embedded in the plasma membrane of the target cell; (ii) the activated receptor in turn results in activation of one or more intracellular signaling pathways involving a series of signaling proteins; and finally (iii) one or more of the intracellular signaling proteins distribute the signal to the appropriate intracellular targets. The targets that lie at the end of signaling pathways are known as effector proteins, which are altered in some way by the incoming signal and implement the appropriate changes in cell behavior. The effector proteins can be transcription regulators, ion channels, metabolic enzymes, and cytoskeletal components.

\subsubsection{Cell Signaling: Second Messengers and Molecular Switches-Most} intracellular signaling molecules are proteins which help relay the signal into the cell by either generating second messengers, or activating the next signaling or effector protein in the pathway. Second messengers are small chemicals (e.g., cyclic AMP, $\mathrm{Ca}^{2+}$, and diacylglycerol) that are generated upon receptor activation by the extracellular stimulus (also known as extracellular signaling molecule or first messenger) and diffuse away from their source, spreading the signal to other parts of the cell.

Many of the intracellular signaling proteins behave like molecular switches, which are typically controlled or regulated by phosphorylation and dephosphorylation catalyzed by selective protein kinases and phosphatases, respectively. Although phosphorylation/ dephosphorylation plays a predominant role in cell signaling, as described next, substantial 
evidence also suggests a critical role for ROS-mediated redox reactions in cell signal transduction under certain conditions.

5.3.3. Redox Signaling: Definitions-As noted earlier, due to the presence of various antioxidant defenses, small transient increases in ROS levels are unlikely to cause significant injury to cells. Instead, such transient increases in ROS levels can fulfill a signaling role, resulting in physiological responses. The term redox signaling refers to the process wherein ROS or related reactive species act as second messengers to cause physiological cellular responses via redox reactions. In this context, ROS can cause redox modulation of signaling proteins, such as protein kinases and transcription factors to lead to physiological cellular responses. These responses may include cell proliferation and differentiation, as well as altered production or expression of cellular products, such as cytokine and adhesion molecules. It is necessary to emphasize the difference between redox signaling and redox modulation. Redox signaling refers to a physiological process, where ROS act as second messengers to mediate responses that are required for proper function and survival of the cell. On the other hand, redox modulation (or redox regulation) refers to a process wherein ROS alter the activity or function of the redox-sensitive molecular targets, including signaling proteins and metabolic enzymes, leading to either physiological or pathophysiological responses. When pathophysiological responses occur it is also known as oxidative stress. Hence, in terms of ROS- mediated redox reactions, redox signaling emphasizes the role of ROS as second messengers (like cyclic AMP or $\mathrm{Ca}^{2+}$ ) to mediate physiological responses, whereas redox modulation (regulation) stresses the role of ROS in altering the redox sensitive targets and that in the process the ROS involved usually do not act as second messengers (Table 2).

\subsubsection{Redox Signaling: The Three Components and an Established Case-}

Cellular redox reactions and their regulation rely on an extensive array of biomolecules or cellular processes to (i) generate the ROS in a regulated manner, (ii) scavenge or inactivate the ROS, and (iii) sense the cellular redox milieu chemically by undergoing oxidation and reduction reactions [19, 20]. Figure 4 illustrates the concept of cellular redox signaling. Notably, a number of cellular processes are known to produce ROS under both physiological and pathophysiological conditions.

The emerging role of ROS as second messengers in cell signal transduction has broadened the concept of cell signaling by providing a novel mechanism for cells to respond to their environment. While the area of redox signaling has been rapidly evolving over the past few years, the concept that ROS acting as second messengers in cell signaling originated from the early work on nitric oxide. In this regard, the role of nitric oxide (a free radical), acting as a second messenger, in cell signal transduction is a well-established concept [12]. In fact, the Nobel Prize in Physiology or Medicine for 1998 was awarded to Robert F. Furchgott, Louis J. Ignarro and Ferid Murad for their discoveries made in the 1980s, concerning nitric oxide as a signaling molecule in the cardiovascular system. 


\section{ROS PARADIGM}

ROS paradigm (Figure 5) addresses the following aspects: (i) sources (generation) of free radicals and related species, especially ROS; (ii) molecular interactions between ROS and molecular targets in the biological systems; (iii) the resulting pathophysiological consequences; and (iv) the resulting physiological responses. This paradigm also emphasizes the impact of antioxidants on the above processes and provides a framework for guiding antioxidant-based intervention of human diseases.

\subsection{Sources of ROS}

ROS are formed from various endogenous sources, including mitochondrial electron transport chain and $\mathrm{NAD}(\mathrm{P}) \mathrm{H}$ oxidases. They are also derived from exogenous sources, such as radiation, air pollutants, and certain xenobiotics that undergo continuous reduction and oxidation cycles, i.e., redox cycling. The levels of ROS in a biological system are determined not only by the rates of their production, but also by the presence and activities of cellular antioxidant defenses. Electrophiles (see Section 2.6) that deplete cellular GSH can lead to secondary oxidative stress. In this regard, a number of environmental chemicals as well as drugs are metabolized to form electrophilic metabolites.

\subsection{Molecular Interactions between ROS and Cellular Targets}

ROS are reactive species that cause oxidative damage to cellular biomolecules, including proteins, lipids, and nucleic acids. As such, mammalian cells are equipped with a wide variety of antioxidants and other cytoprotective factors to protect them from damage by ROS. On the other hand, due to their ability to react with redox sensitive signaling proteins, under physiological conditions, ROS can also act as second messengers to participate in cell signal transduction, a process known as redox signaling. The coordination between ROS generation and antioxidant-mediated decomposition ensures that ROS levels are tightly controlled and fine-tuned so as to act as second messengers for cell signaling.

\subsection{Pathophysiological Consequences}

When ROS overwhelm the normal cellular or tissue defenses, oxidative stress ensures, leading to pathophysiological processes. Indeed, oxidative stress is an important pathophysiological mechanism underlying various human diseases. In this regard, it is conceivable that decreasing the levels of ROS or inhibiting the oxidative damage through using antioxidant-based strategies may provide significant beneficial effects.

\subsection{Physiological Responses}

ROS also play important physiological roles. One most widely known scenario in this regard is the production of ROS from phagocytic cells in killing invading pathogenic microorganisms. Production of ROS by phagocytic cells is recognized as an important part of the innate immunity. As described above, ROS also act as second messengers to mediate cell redox signaling, leading to physiological responses. Therefore, ROS also fulfill important physiological functions. It is conceivable that interference with the above physiological functions of ROS by antioxidants may cause undesired effects in biological systems. In this regard, uncontrolled overexpression of $\mathrm{Nrf2}$, a master regulator of 
antioxidant genes, promotes tumorigenesis [21]. In addition, ROS are also involved in drug action. For example, metabolism of certain anticancer drugs leads to formation of ROS, which in turn mediates cancer cell killing [22]. Recently, targeting ROS to cancer cells has been developed as a potentially promising strategy for cancer therapy [23].

\subsection{ROS Paradigm-Guided Antioxidant-Based Intervention}

When examining the biological effects of ROS, both detrimental and beneficial effects of these species should be taken into consideration. The ROS paradigm defines the scope of ROS biology and medicine. The goals of ROS biology and medicine should include not only determination of the pathophysiological role of ROS in diseases, but also investigation of the physiological functions of these reactive species in biological systems. Likewise, antioxidant-based strategies for disease intervention should be devised to selectively control the adverse effects caused by pathophysiological levels of ROS without compromising the beneficial effects of these reactive species at physiological levels. Thus, one has to be cautious about the overuse of antioxidants or antioxidant supplements, which appear to be over consumed.

\section{Acknowledgments}

The work was supported in part by a grant from the U.S. National Institutes of Health/National Cancer Institute (CA192936).

\section{ABBREVIATIONS}

$\begin{array}{ll}\text { GSH } & \text { reduced form of glutathione } \\ \text { GSSG } & \text { oxidized form of glutathione } \\ \text { ROS } & \text { reactive oxygen species } \\ \text { RNS } & \text { reactive nitrogen species }\end{array}$

\section{References}

1. Halliwell B. Antioxidants in human health and disease. Annu Rev Nutr. 1996; 16:33-50. DOI: 10.1146/annurev.nu.16.070196.000341 [PubMed: 8839918]

2. Trush MA, Seed JL, Kensler TW. Oxidant-dependent metabolic activation of polycyclic aromatic hydrocarbons by phorbol ester-stimulated human polymorphonuclear leukocytes: possible link between inflammation and cancer. Proc Natl Acad Sci USA. 1985; 82(15):5194-8. [PubMed: 2991910]

3. Thompson DC, Cha YN, Trush MA. The peroxidase-dependent activation of butylated hydroxyanisole and butylated hydroxytoluene (BHT) to reactive intermediates: formation of BHTquinone methide via a chemical-chemical interaction. J Biol Chem. 1989; 264(7):3957-65. [PubMed: 2492993]

4. Trevisani M, Siemens J, Materazzi S, Bautista DM, Nassini R, Campi B, et al. 4-Hydroxynonenal, an endogenous aldehyde, causes pain and neurogenic inflammation through activation of the irritant receptor TRPA1. Proc Natl Acad Sci USA. 2007; 104(33):13519-24. DOI: 10.1073/pnas. 0705923104 [PubMed: 17684094]

5. Ruef J, Rao GN, Li F, Bode C, Patterson C, Bhatnagar A, et al. Induction of rat aortic smooth muscle cell growth by the lipid peroxidation product 4-hydroxy-2-nonenal. Circulation. 1998; 97(11):1071-8. [PubMed: 9531254] 
6. Sawa T, Zaki MH, Okamoto T, Akuta T, Tokutomi Y, Kim-Mitsuyama S, et al. Protein Sguanylation by the biological signal 8-nitroguanosine $3^{\prime}, 5^{\prime}$-cyclic monophosphate. Nat Chem Biol. 2007; 3(11):727-35. DOI: 10.1038/nchembio.2007.33 [PubMed: 17906641]

7. Ito C, Saito Y, Nozawa T, Fujii S, Sawa T, Inoue H, et al. Endogenous nitrated nucleotide is a key mediator of autophagy and innate defense against bacteria. Mol Cell. 2013; 52(6):794-804. DOI: 10.1016/j.molcel.2013.10.024 [PubMed: 24268578]

8. Talalay P. Chemoprotection against cancer by induction of phase 2 enzymes. Biofactors. 2000; 12(1-4):5-11. [PubMed: 11216505]

9. Noubade R, Wong K, Ota N, Rutz S, Eidenschenk C, Valdez PA, et al. NRROS negatively regulates reactive oxygen species during host defence and autoimmunity. Nature. 2014; 509(7499):235-9. DOI: 10.1038/nature13152 [PubMed: 24739962]

10. Zhang K, Kaufman RJ. From endoplasmic-reticulum stress to the inflammatory response. Nature. 2008; 454(7203):455-62. DOI: 10.1038/nature07203 [PubMed: 18650916]

11. Zhou R, Yazdi AS, Menu P, Tschopp J. A role for mitochondria in NLRP3 inflammasome activation. Nature. 2011; 469(7329):221-5. DOI: 10.1038/nature09663 [PubMed: 21124315]

12. Roth TL, Nayak D, Atanasijevic T, Koretsky AP, Latour LL, McGavern DB. Transcranial amelioration of inflammation and cell death after brain injury. Nature. 2014; 505(7482):223-8. DOI: 10.1038/nature12808 [PubMed: 24317693]

13. Keleku-Lukwete N, Suzuki M, Otsuki A, Tsuchida K, Katayama S, Hayashi M, et al. Amelioration of inflammation and tissue damage in sickle cell model mice by Nrf2 activation. Proc Natl Acad Sci USA. 2015; 112(39):12169-74. DOI: 10.1073/pnas.1509158112 [PubMed: 26371321] 13a Sies H. Oxidative stress: a concept in redox biology and medicine. Redox Biol. 2015; 4:180-3. DOI: 10.1016/j.redox.2015.01.002 [PubMed: 25588755]

14. Radi R. Peroxynitrite, a stealthy biological oxidant. J Biol Chem. 2013; 288(37):26464-72. DOI: 10.1074/jbc.R113.472936 [PubMed: 23861390]

15. Szabo C, Ischiropoulos H, Radi R. Peroxynitrite: biochemistry, pathophysiology and development of therapeutics. Nat Rev Drug Discov. 2007; 6(8):662-80. DOI: 10.1038/nrd2222 [PubMed: 17667957]

16. Hess DT, Matsumoto A, Kim SO, Marshall HE, Stamler JS. Protein S-nitrosylation: purview and parameters. Nat Rev Mol Cell Biol. 2005; 6(2):150-66. doi: nrm1569 [pii] 10.1038/nrm1569. [PubMed: 15688001]

17. Yun BW, Feechan A, Yin M, Saidi NB, Le Bihan T, Yu M, et al. S-nitrosylation of NADPH oxidase regulates cell death in plant immunity. Nature. 2011; 478(7368):264-8. DOI: 10.1038/ nature10427 [PubMed: 21964330]

18. Go YM, Jones DP. The redox proteome. J Biol Chem. 2013; 288(37):26512-20. DOI: 10.1074/ jbc.R113.464131 [PubMed: 23861437]

19. Rudolph TK, Freeman BA. Transduction of redox signaling by electrophile-protein reactions. Sci Signal. 2009; 2(90):re7.doi: 10.1126/scisignal.290re7 [PubMed: 19797270]

20. Kawagishi H, Finkel T. Unraveling the truth about antioxidants: ROS and disease: finding the right balance. Nat Med. 2014; 20(7):711-3. DOI: 10.1038/nm.3625 [PubMed: 24999942]

21. DeNicola GM, Karreth FA, Humpton TJ, Gopinathan A, Wei C, Frese K, et al. Oncogene-induced Nrf2 transcription promotes ROS detoxification and tumorigenesis. Nature. 2011; 475(7354):1069. DOI: 10.1038/nature10189 [PubMed: 21734707]

22. Trush MA, Mimnaugh EG, Gram TE. Activation of pharmacologic agents to radical intermediates: implications for the role of free radicals in drug action and toxicity. Biochem Pharmacol. 1982; 31(21):3335-46. [PubMed: 6756414]

23. Trachootham D, Alexandre J, Huang P. Targeting cancer cells by ROS-mediated mechanisms: a radical therapeutic approach? Nat Rev Drug Discov. 2009; 8(7):579-91. DOI: 10.1038/nrd2803 [PubMed: 19478820] 


\section{SUMMARY OF KEY POINTS}

- $\quad$ ROS biology and medicine may be defined as a field that studies the biological effects of ROS and related species as well as antioxidants with an emphasis on the involvement of these molecular entities in health and disease.

- $\quad$ ROS is a collective term to include superoxide, hydrogen peroxide, hydroxyl radical, singlet oxygen, peroxyl radical, alkoxyl radical, lipid hydroperoxide, peroxynitrite, hypochlorous acid, and ozone. Some ROS, such as superoxide and hydroxyl radicals are free radicals; others such as hydrogen peroxide and peroxynitrite are non-radical ROS, but can give rise to free radicals upon further metabolisms in biological systems.

- The term RNS has been coined to include nitric oxide, peroxynitrite, nitrogen dioxide radical, and other oxides of nitrogen or nitrogen-containing reactive species. Because RNS are almost exclusively oxygen-containing reactive species, by definition, they may also be considered as ROS.

- Due to the potential harmful effects of ROS, aerobic organisms have evolved a series of antioxidant defenses to protect vital biomolecules from ROSmediated damage. The term antioxidant refers to any substance that can prevent, reduce, or repair the ROS-induced damage of a target biomolecule at physiologically relevant concentrations.

- As ROS and inflammation are intimately intertwined, many antioxidants also possess anti-inflammatory activities. Hence, the compound term 'antioxidative/anti-inflammatory defenses' is sometimes used to describe these antioxidants.

- Under physiological conditions, cellular ROS and antioxidants are in balance, thereby ensuring physiological homeostasis. Oxidative stress refers to a condition where the levels of ROS significantly overwhelm the capacity of antioxidant defenses in a biological system. Moderate oxidative stress causes cell dysfunction and altered behavior, whereas overt oxidative stress usually induces cell death.

- Under certain conditions, ROS act as second messengers to participate in cell signal transduction. The concept of cell signaling defines the ability of cells to detect changes in their environment to generate an appropriate physiological response upon information processing. The term cellular redox signaling refers to the process wherein ROS or related species act as second messengers to cause cellular responses via redox reactions between ROS and signaling proteins.

- It is necessary to distinguish redox signaling from redox modulation/ regulation. Redox signaling emphasizes a physiological process, wherein ROS act as second messengers to mediate responses that are required for proper function and survival of the cell. On the other hand, redox modulation/ regulation refers to a process wherein ROS alter the activity/function of the 
redox-sensitive molecular targets, including signaling proteins and metabolic enzymes, leading to physiological or pathophysiological responses.

- $\quad$ ROS paradigm defines the scope of ROS biology and medicine, and thereby provides a framework for understanding the involvement of ROS in health and disease and for guiding antioxidant-based strategies to selectively control ROS-induced damage without compromising the physiological functions of these species. 


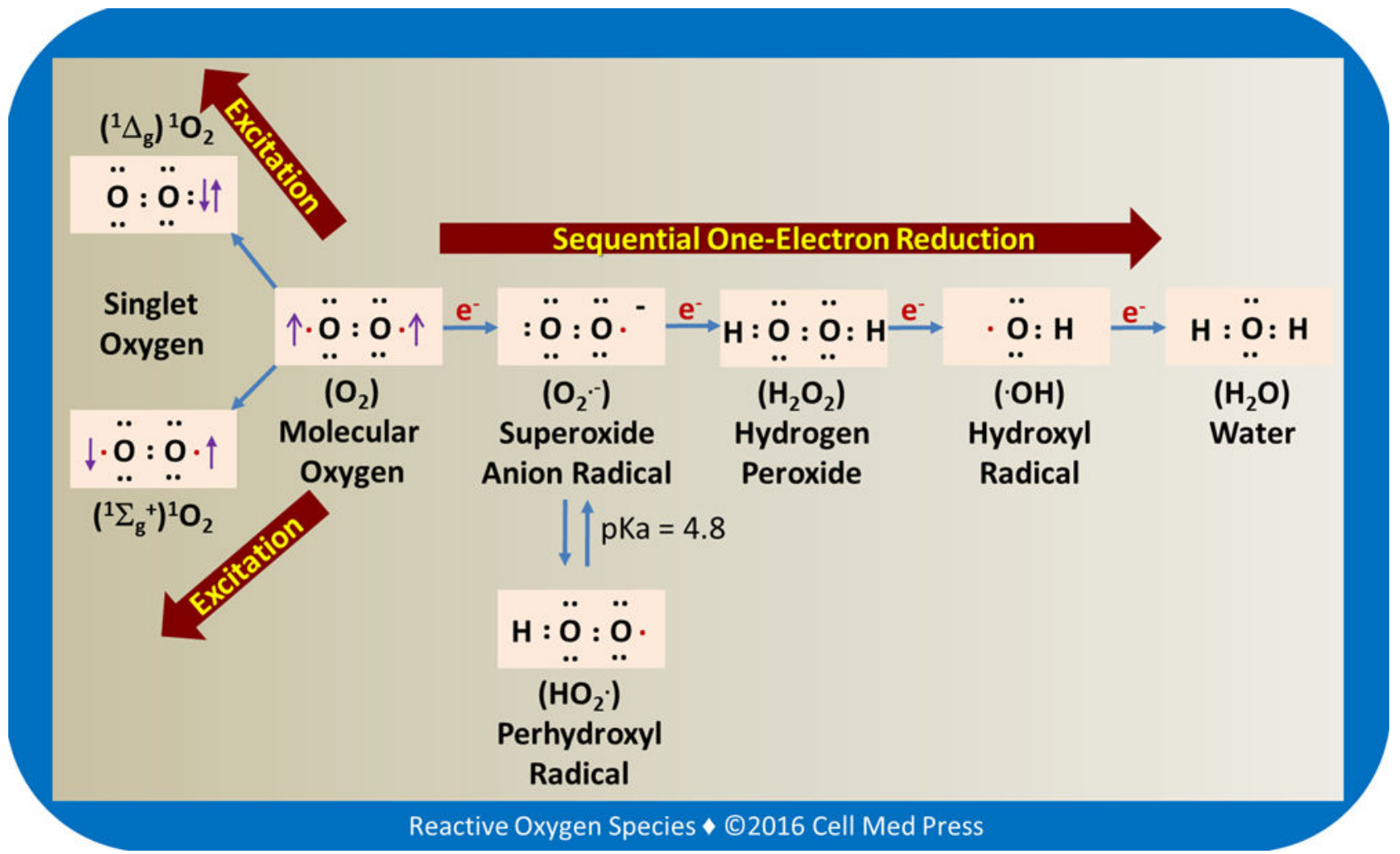

FIGURE 1. Excitation and univalent reduction of molecular oxygen to yield reactive oxygen species (ROS) in biological systems

As indicated, ground state molecular oxygen $\left(\mathrm{O}_{2}\right)$ is a free radical (actually a di-radical) because it contains two unpaired electrons. $\mathrm{O}_{2}$ is much less reactive than ROS due to spin restriction caused by the same spin direction of its two unpaired electrons. $\mathrm{O}_{2}$ can be excited to form singlet oxygen $\left({ }^{1} \mathrm{O}_{2}\right)$. There are two states of singlet oxygen: delta and sigma. The sigma state singlet oxygen is a free radical, whereas the delta state is a non-radical. One electron reduction of $\mathrm{O}_{2}$ gives rise to superoxide anion radical $\left(\mathrm{O}_{2}{ }^{\mathbf{-}}\right)$, which then undergoes another one electron reduction to yield hydrogen peroxide $\left(\mathrm{H}_{2} \mathrm{O}_{2}\right)$. One electron reduction of hydrogen peroxide generates hydroxyl radical $\left(\mathrm{OH}^{\mathbf{*}}\right)$, which can then be reduced by one electron to form water. Perhydroxyl radical $\left(\mathrm{HO}_{2}\right)$ is a protonated form of superoxide anion radical. 


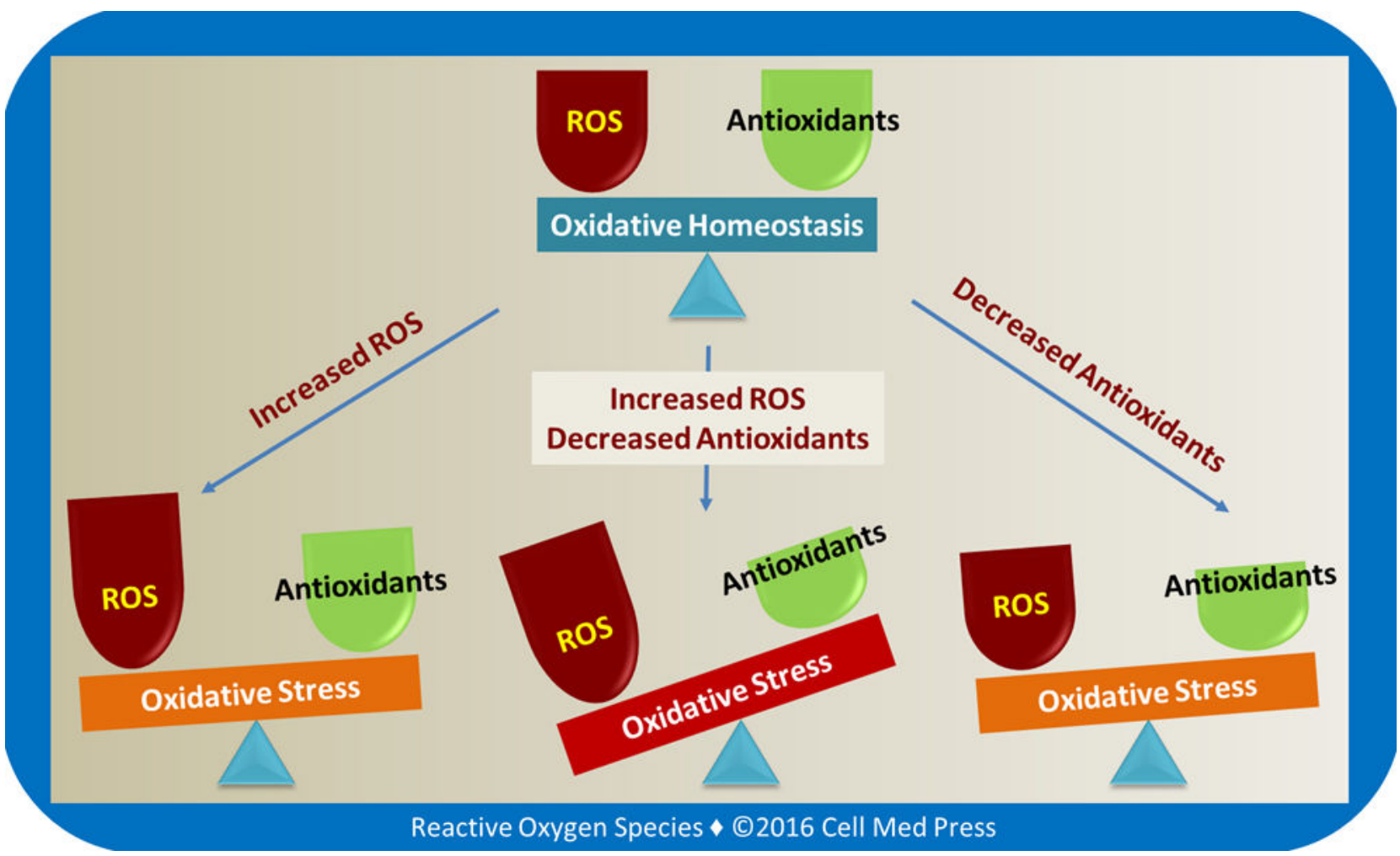

FIGURE 2. Schematic illustration of oxidative stress

As depicted, oxidative stress is caused by either increased formation of reactive oxygen species (ROS) or decreased antioxidant defenses, or both. It is important to distinguish oxidative stress from redox signaling. Oxidative stress emphasizes the potential detrimental effects of increased ROS, whereas redox signaling underlines the involvement of ROS in cell signaling transduction leading to physiological responses. 


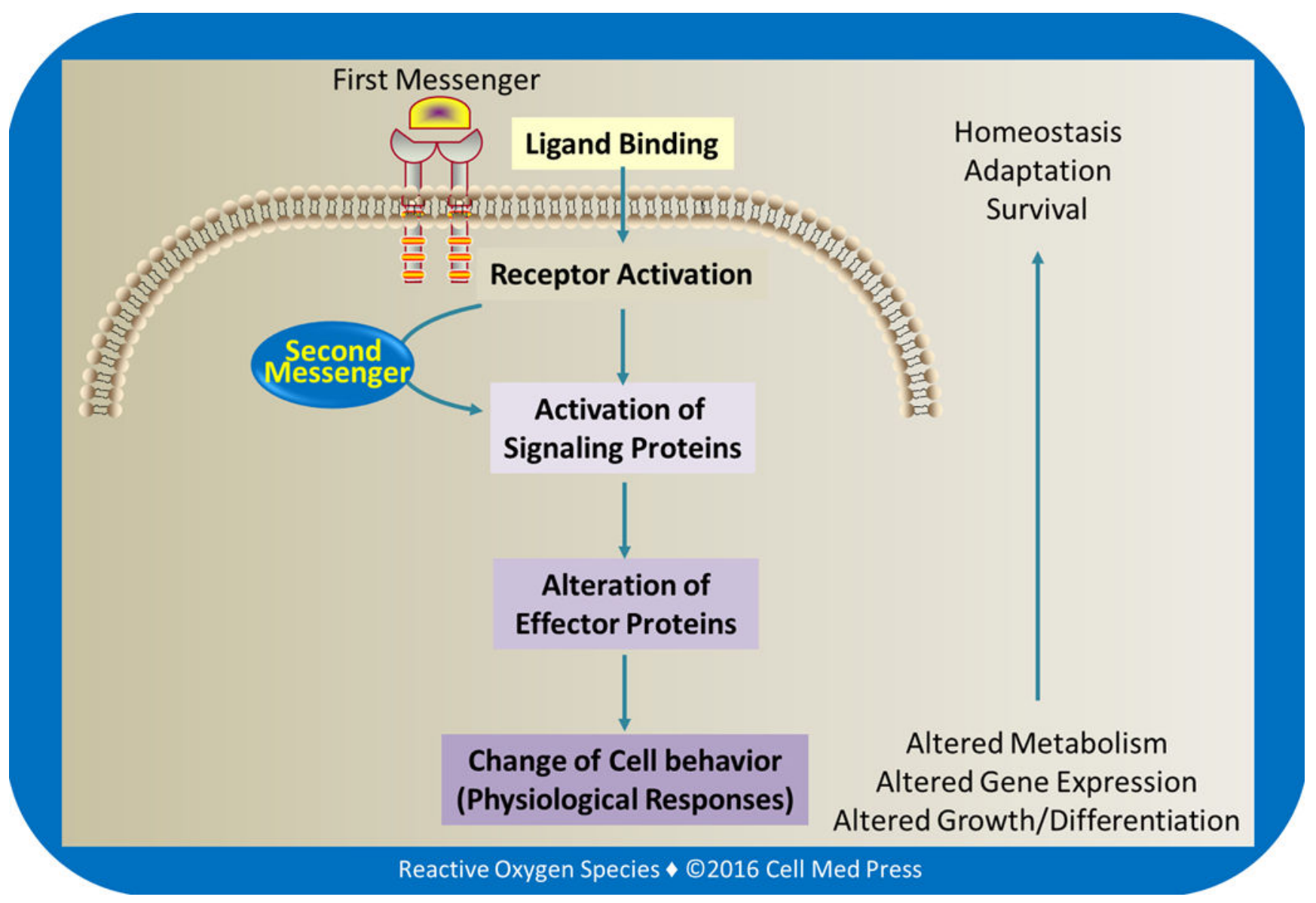

FIGURE 3. The three major steps of cell signaling

This scheme illustrates the three major steps involved in cell signal transduction upon extracellular stimulation. The extracellular stimulus (signaling molecule) can be a growth factor that acts on the target cell to cause a physiological cellular response (e.g., cell proliferation). The entire signal transduction process consists of three major steps: (i) binding of the extracellular signaling molecule to its receptor embedded in the plasma membrane of the target cell, leading to receptor activation; (ii) the activated receptor in turn either directly or indirectly (via formation of second messengers, such as cyclic AMP) causes activation of the signaling molecules (typically proteins) of one or more of the signal transduction pathways; and finally (iii) one or more of the activated signaling proteins alter the activity of effector proteins that reside at the end of signaling pathways and thereby the behavior of the cell. 


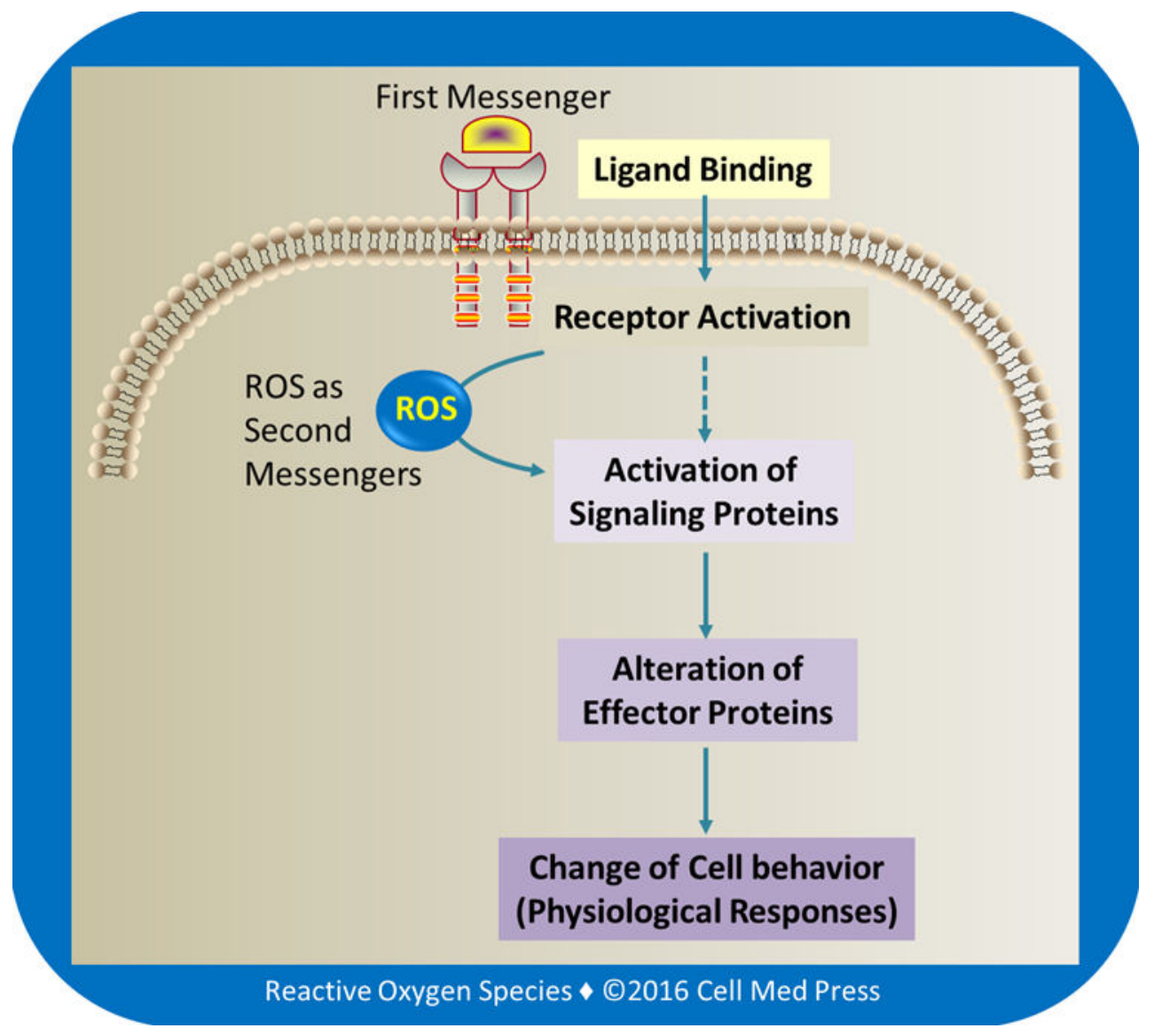

FIGURE 4. Components of reactive oxygen species (ROS)-mediated redox signaling ROS- mediated cellular redox signaling involves multiple components. The generators are cellular processes (e.g., mitochondrial respiration and activation of NAD(P)H oxidases) responsible for the controlled production of the ROS, whereas the terminators (e.g., antioxidants) act to scavenge or inactivate the ROS so that the formation and disappearance of the ROS occur in a regulated manner. The sensor molecules (e.g., protein kinases, transcription factors, or other proteins) sense the ROS-induced changes of the cellular redox milieu chemically by undergoing oxidation and reduction reactions. Such redox reactions modulate the functions or conformations of the sensors, altering activities of downstream effectors, leading to cellular responses. Also see text (Section 5.3) for additional description. 


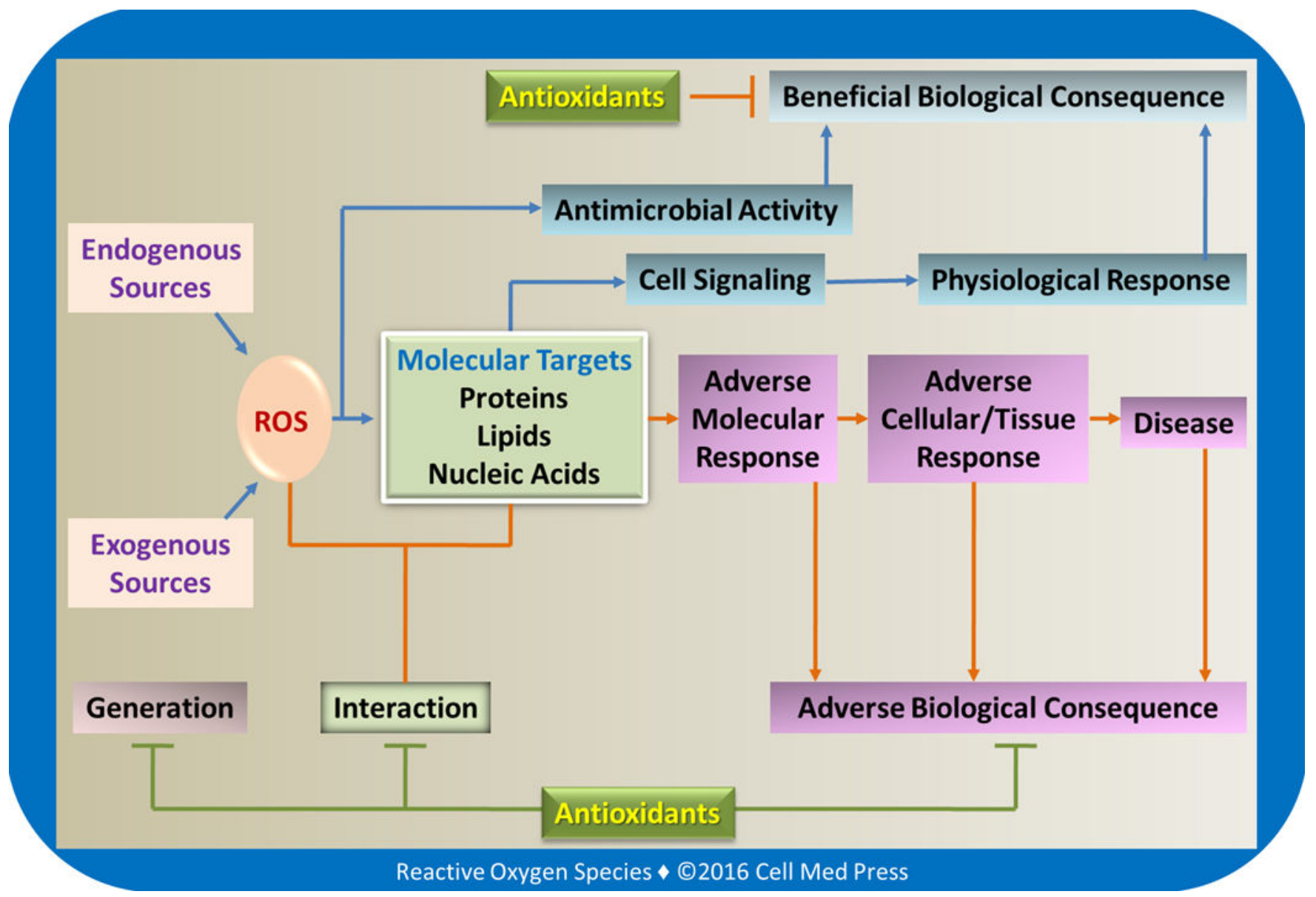

FIGURE 5. The ROS paradigm

This paradigm defines the scope of ROS biology and medicine which includes generation of ROS and related reactive species, the interactions of these reactive species with target biomolecules, and the resulting biological consequences (adverse or beneficial). This paradigm also depicts the antioxidant-based intervention of diseases associated with ROS, emphasizing the importance of selectively controlling ROS-mediated adverse effects without compromising the physiological functions of these species. 


\section{TABLE 1}

The number of entries in PubMed involving the use of 'reactive oxygen species' and other related terms in title/abstract

\begin{tabular}{|ll|}
\hline Term & Entries $^{\boldsymbol{a}}$ \\
\hline Reactive oxygen species & 74,134 \\
\hline Reactive oxygen intermediates & 1,860 \\
\hline Reactive oxygen metabolites & 1,113 \\
\hline Oxygen radicals & 5,027 \\
\hline Reactive nitrogen species & 2,047 \\
\hline Reactive chlorine species & 25 \\
\hline
\end{tabular}

Note:

${ }^{a}$, number of entries as of December 9, 2015 


\section{TABLE 2}

\section{Differences between ROS-mediated redox signaling and redox modulation/regulation}

\begin{tabular}{|lll|}
\hline Characteristic & Redox Signaling & Redox Modulation/Regulation \\
\hline $\begin{array}{l}\text { Dependence of ROS formation on } \\
\text { "first messengers" }\end{array}$ & $\begin{array}{l}\text { Dependent on "first messengers"; always } \\
\text { produced by the target cells through a well- } \\
\text { defined cellular mechanism and under tight } \\
\text { control }\end{array}$ & $\begin{array}{l}\text { Independent of "first messengers"; may be produced by } \\
\text { the target cells or adjacent cells or from environmental } \\
\text { sources }\end{array}$ \\
\hline ROS as second messengers & No & Yes \\
\hline ROS targets & Signaling proteins only & Signaling proteins; metabolic enzymes; others \\
\hline Always involving cell signaling & No & Yes \\
\hline Nature of cellular responses & Physiological & Physiological or pathophysiological \\
\hline $\begin{array}{l}\text { Oxidative stress as a possible } \\
\text { outcome }\end{array}$ & No & Yes \\
\hline
\end{tabular}

